\title{
THE ACTION OF SOME ENZYMES ON MILK-SUGAR.
}

By H. Droop Richmond.

(Read at the Meeting on October 5th, 1892.)

THese short notes formed part of an investigation of the methods for the complete analysis of milk and milk products. They were originally intended to see if rennet had any action on milk-sugar; it seemed very unlikely that this was the case, but being unable to find any experiments actually proving this, I decided to try for myself. I was also led to investigate this on account of Pappel and myself having isolated from gamoose milk a sugar, towfi'ose (tewfikose) by a method which avoided the use of rennet, and it appeared just possible that milk sugar might be formed from some compound existing in the milk by the action of rennet. 
I assume that all sugars having the same rotary and reducing powers as milk-sugar are that compound, and that if the rotary and reducing powers do not alter, that the milk-sugar is unchanged. This assumption, I take it, will be granted by all.

The polariscope used was the Mitscherlich half-shadow polariscope described by Vieth (ANALYsT, xi. 141); it is not a very delicate instrument, but I find that I can read to $.025^{\circ}$, by taking the average of several (say 10) readings. In the paper referred to Vieth states that the readings may be taken as giving grams of milk-sugar in 100 c.c. This, I find, is not quite, though nearly accurate. The tube used has a length of 198.2 millimetres, and the scale is divided into angular degrees; taking the specific rotary power $[\boldsymbol{a}]_{\mathrm{o}}$ of milk-sugar to be $52.5^{\circ}$ (c.f. Wiley, Amer. Chem. $J$, vi. No. 5) the readings

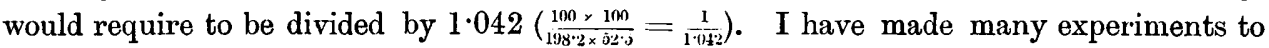
determine the actual factor, and find that the readings require to be divided by 1.045 to give grams per 100 c.c. These two factors agree well within the limit of reading.

I find, on the examination of the published statements as to the reducing power of milk-sugar, that some diversity of opinion occurs as to the method of obtaining accurate results. Thus, Soxhlet (J.f. Prakt. Chem. [2] xxi. 277, et seq.) finds that the same results are obtained whether the Fehling's solution be diluted or not; the conclusion of Rodewald and Tollens (Ber., xi. 2076) does not quite accord with Soxhlet's. Muter (ANALYsT, v. 35) indicates that, in order to ensure constant results, the sugar must be diluted till the solution contain less than 1 gram in 100 c.c., and a very slight excess of Fehling's solution must be added. Jones (ANalyst, xiv. 81) employs O'Sullivan's method, in which a large excess of Fehling's solution, diluted with two measures of water, are employed, and brought to the temperature of the water-bath before the sugar is added, and the heating is continued for 14 minutes.

I have made some experiments on this subject, and find that O'Sullivan's method gives the most concordant results. I also find that, if the Fehling's solution is diluted with an equal bulk of water, I obtain the same results as when diluting with two volumes of water, and there is less solution to be filtered. I collect the precipitate of $\mathrm{Ca}_{2} \mathrm{O}$ on a filter, and burn first over a bunsen and then in a muffle. Duplicates do not differ more than about 1 milligram. I find that the filter absorbs 2 milligrams from the Fehling's solution, and therefore deduct this. Jones gives the factor to convert copper oxide into milk-sugar (anhydrous) as 0.6024 . I myself find 0.6025 , and calculate from the mean of Rodewald and 'Tollens' results the factor 0.6022 .

The method and factor given in the valuable paper of Jones are therefore to be relied upon.

My first experiments were made by taking milk and estimating therein the milksugar, and estimating the milk-sugar in the whey produced by rennet in the same milk, and calculating the proportion of the milk-sugar to 100 parts of water in each. 
The mean of three experiments gave-

$$
\text { Milk-sugar (polarised) }
$$

Milk.

Whey.

" (gravimetric)

Rennet has therefore no action on milk-sugar, and milk-sugar exists in the milk as the same compound which exists in the whey.

I next made a solution of milk-sugar; 25 grams of finely-powdered milk-sugar, which had been very carefully purified by Dr. Vieth, were shaken for five minutes with 450 c.c. of cold water, and immediately filtered, and the solution mixed and polarized. The readings were taken at from 7 to 8 minutes after the addition of the sugar to the water; after a further ten minutes, the readings were again taken; and again at intervals of quarter-hour, half-hour, one hour, six hours, and twenty-fours. Taking the first reading to have been at an interval of five minutes from the mean time of solution, I constructed a time-curve for the fall in rotary power, and from this calculate that at the moment of solution the specific rotary power is $82 \cdot 0 \pm 2$. I also estimated the density of the solution at $15.5^{\circ}$ after an interval of ten minutes, and after an interval of twenty-four hours. In each case it was $1 \cdot 0193$, showing that no change in the density had occurred concurrently with the change in the specific rotary power.

This solution contained 5.20 grams in 100 c.c. of milk-sugar determined by evaporation, 5.22 grams by the polariscope, and 5.18 grams from the weight of the copper oxide reduced, and $5 \cdot 17$ grams from density.

With this solution the following experiments were tried :-

Action of rennet.-To 50 c.c. of this solution 10 milligrams of rennet were added, and this was kept at $40^{\circ} \mathrm{C}$ for one hour. After cooling and filtering, it gave the following :-

$$
\begin{array}{cc}
\text { Sugar (polarised) } & 5 \cdot 22 \\
" \quad \text { (gravimetric) } & 5 \cdot 23
\end{array}
$$

This shows again that rennet has no action on milk-sugar.

Action of pepsin.-To 50 c.c. of this solution 5 c.c. $1 \%$ hydrochloric acid and 03 grams of pepsin were added, and the solution heated for 3 hours at $40^{\circ} \mathrm{C}$. After cooling, filtering, and correction for the small figures obtained in a blank experiment, the following figures were obtained :-

$$
\begin{aligned}
& \text { Sugar (polarised) } \quad \mathbf{5 \bullet 2 4} \\
& \text { " (gravimetric) } \quad 5 \cdot 19
\end{aligned}
$$

Pepsin is without action on milk-sugar.

Action of pancreatic extract.-To 50 c.c. of this solution were added 1 c.c. of pancreatic extract, and 05 grams of sodium bicarbonate, and the whole digested at $55^{\circ} \mathrm{C}$ for 
one hour. After cooling, filtering, and correction for the small figures obtained in a blank experiment, the following figures were obtained:-

$$
\text { Sugar (polarised) }
$$$$
" \text { (gravimetric) }
$$

Pancreatic extract has then no action on milk-sugar.

In the experiments quoted in no case was the enzyme destroyed by heating before estimating the sugar. It was found, in all cases, that while the reducing power was only slightly affected thus, the specific rotary power was diminished (in the case of pepsin digestions the acid being first neutralized). Experiments on milk showed also that the specific rotary power of the sugar is reduced by heating. The study of the changes of milk-sugar by heat is reserved for a future communication.

These experiments show that the milk-sugar in whey and in artificially digested milk can be accurately estimated, provided that they have not been heated.

The change of rotary power on heating milk-sugar (in solution) shows that some chemical change is taking place. This sheds some light on the fact that constant results cannot be obtained on continued drying of the total solids of milk unless the water is rapidly evaporated. I have noticed that if precautions are taken to get rid of the bulk of the water in a short time (c.f.ANALYsT, xiv. 121) that the rasidue does not turn brown on continued heating. As in ordinary total solid estimation about one hour elapses before the residue is apparently dry, there is sufficient time allowed for considerable decomposition, which may (and probably does) render the results inaccurate. On this point I believe that my investigation of the changes of milk-sugar on heating will shed considerable light.

[Note.-With reference to the slight error involved by taking the reading of the Mitscherlich half-shadow polariscope to indicate grams of milk-sugar per 100 c.c., Dr. Vieth writes me that he has long been aware that it is not correct; but as the error introduced is small, he has preferred to call the actual readings milk-sugar on account of practical convenience. The mean of Dr. Vieth's readings on accurately prepared solutions of milk-sugar gives the factor 1.044.-H. D. R.] 